\title{
Mitral Valve Replacement and Subaortic Membrane Resection Following Pneumonectomy
}

\author{
Melih Hulusi Us, Murat Ugurlucan, Murat Basaran, Ozer Selimoglu, and Ali Kocailik \\ Department of Cardiovascular Surgery, Goztepe Safak Hospital, Istanbul 34000, Turkey \\ Correspondence should be addressed to Murat Ugurlucan, muratugurlucan@yahoo.com
}

Received 19 December 2009; Accepted 5 January 2010

Academic Editor: Michael S. Firstenberg

Copyright (๑) 2010 Melih Hulusi Us et al. This is an open access article distributed under the Creative Commons Attribution License, which permits unrestricted use, distribution, and reproduction in any medium, provided the original work is properly cited.

\begin{abstract}
The pulmonary status is a vital factor for patients undergoing open heart surgery. The cardiac surgery itself deteriorates the actual pulmonary functions. Today, patients are no longer living with a cardiac disease due to compromised respiratory functions secondary to various pathologies, patients with lung disorders more often seek solutions for their cardiac disease and they are commonly operated. However, the resection of a lobe or a whole lung is a major challenge for the patients planned for cardiac surgery. In this report, we present a 65 -year-old patient, who had left pnemonectomy which had been performed 8 years ago and was admitted for mitral valve replacement and subaortic membrane resection.
\end{abstract}

\section{Introduction}

Open heart surgery is associated with postoperative deteriorated respiratory functions. Cardiac surgery is a challenge both for the patients and the whole cardiac surgery and reanimation team in the pre- and postoperative periods as well as during the surgery. The medical literature contains series on concomitant heart and lung surgery [1,2]; however, it lacks a defined consensus for patients who have previously undergone pulmonary resection and who are planned for a cardiac surgery [2-7].

This report presents a 65 -year-old male patient with 8 years history of pneumonectomy and who was operated for left ventricular outflow tract stenosis and mitral valve disease.

\section{Case Report}

A 65-year-old male patient presented to the clinic with dyspnea and easy fatigability. In his medical record there was a left pneumonectomy which was performed 8 years ago. The physical examination revealed normal temperature, blood pressure but atrial fibrillation. There was mid-late systolic murmur on cardiac auscultation. No respiratory sounds could be heard in the left hemithorax and right side lung sounds were normal. The chest X-ray examination indicated deviation of the mediastinal structures to the left and hyperinflation of the right lung (Figure 1). On echocardiography, there were severe mitral regurgitation and mitral stenosis most probably due to rheumatic origin (mean gradient of $10 \mathrm{mmHg}$ with mitral valve area of $1.7 \mathrm{~cm}^{2}$ ), dilated left atrium $(7.8 \times 8.5 \mathrm{~cm}$ diameters $)$, moderate tricuspid valve insufficiency $(2-3+)$, and mild aortic regurgitation and a subaortic ridge with a mean aortic gradient of $25 \mathrm{mmHg}$, and ejection fraction of 55\%. The pulmonary function tests showed a mixed obstructive and restrictive pattern with forced expiratory volume in 1 second and forced expiratory volume at 1 second/forced vital capacity of $1.30 \mathrm{~L}(45 \%$ of the predicted value) and 0.60 (50\% of the predicted value), respectively. The room air arterial blood gas analysis revealed $\mathrm{PaO}_{2}$ of $60 \mathrm{mmHg}, \mathrm{PaCO}_{2}$ of $48 \mathrm{mmHg}$ and oxygen saturation of $90 \%$. After a week of pulmonary rehabilitation with chest physiotherapy, steroids, and bronchodilatators [4], the patient was scheduled for mitral valve replacement and subaortic membrane resection.

Following standard median sternotomy, aortic, and bicaval cannulations, cardiac arrest was provided with antegrade cold blood cardioplegia. Topical ice slush was avoided to minimize the risk of phrenic nerve injury. The mitral valve was replaced with transseptal approach with a $29 \mathrm{~mm}$ St. Jude Medical Mechanical Heart Valve (St. Jude Medical, 


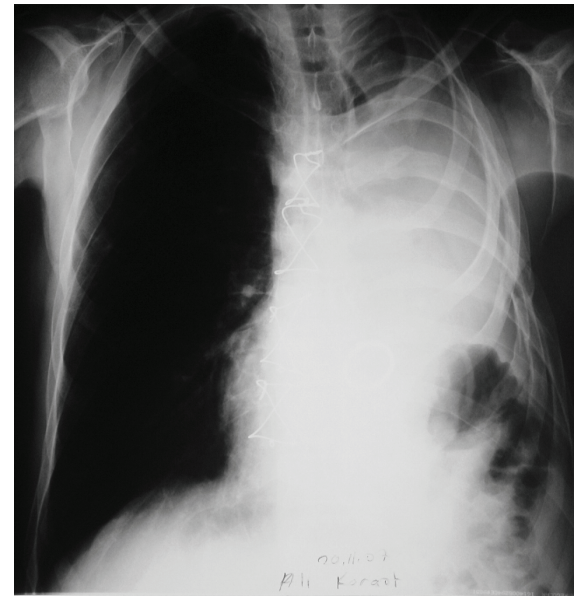

FIGURE 1: Chest roentgenogram of the patient postoperatively. Note the hyperinflated right lung with leftward displacement of the mediastinal structures.

Inc.; St. Paul, Minn, USA). The subaortic fibromuscular membrane together with septal muscular resection was performed through aortotomy. Cross clamp and cardiopulmonary bypass times were 52 and 70 minutes, respectively. The patient was weaned off cardiopulmonary bypass with negative total fluid balance and on $5 \mu \mathrm{gr} / \mathrm{kg} / \mathrm{minute}$ of dobutamine and $3 \mu \mathrm{gr} / \mathrm{kg} / \mathrm{minute}$ of dopamine. At the intensive care unit, forced diuresis was achieved with furosemide to avoid pulmonary edema. Intravenous morphine sulphate was applied near extubation to minimize the pain induced respiratory dysfunction and the patient was extubated at the 6 th postoperative hour. The inotropes were ceased on day 1 after the patient was discharged to the ward. The chest physiotherapy, steroids, and bronchodilatators were continued until the discharge on the 7 th postoperative day. The patient has been regularly followed at our institution since the operation and is free off symptoms 6 months postoperatively.

\section{Discussion}

The preoperative respiratory reserve is an important prognostic factor for patients undergoing open cardiac surgery; apart such procedures themselves are associated with respiratory compromise, atelectasis, and infections [1-5]. Except the emergent cases, cardiac surgery is planned in patients with optimal pulmonary conditions after lung rehabilitation therapy which may be performed according to the variable institutional pulmonology protocols [4]. However, it may not be possible in every case to achieve the optimal conditions prior to surgery. Chronic obstructive pulmonary patterns or lung cancer requiring surgery are frequently encountered in patients with heart problems. These patients are managed concomitantly during cardiac surgery or afterwards $[1,2]$. On the other hand, patients with previous pulmonary operations and more extreme patients such as patients with pneumonectomy constitute a very small fraction of open heart operation candidates [3-7].
A number of issues have been raised in patients undergoing cardiac surgery with prior pneumonectomy. These mainly include maneuvers to prevent decreasing the present pulmonary status. The avoidance of fluid overload preoperatively is of utmost important in these patients with borderline respiratory reserve as well as taking precautions in order to protect the phrenic nerve during the mediastinal dissection and the internal thoracic artery harvesting in case of coronary artery bypass procedures [2-7]. We avoided topical cold ice slush application. The chest rehabilitation therapy with steroids in addition to bronchodilatators may contribute to the decrease in the inflammatory response to cardiopulmonary bypass by reducing the proinflammatory cytokines and increasing the interleukin-10 levels [8].

In a recent review by Stoller et al. [7], a series of 19 patients undergoing cardiac surgery with prior pneumonectomy included 4 patients who had valve replacement. In the series, the most common perioperative complication was pneumothorax [7]. In our patient, we preferred the left internal jugular vein for insertion of the pulmonary artery catheter to avoid a possible pneumothorax at the nonpneumonectomy side.

As the exercise capacity of a patient with prior pneumonectomy and mitral regurgitation is poor, the patient is early symptomatic even when the regurgitation is not severe and the conventional cardiac surgery guidelines may need to be adapted on an individual basis [7]. The exposure of the heart in these patients is another challenge due to the leftward displacement of the mediastinal structures. In addition, the base of the heart is usually displaced posteriorly, hence the mitral valve annulus is difficult to reach through either transseptal or thansatrial approaches [4]. In our patient, we preferred a transseptal approach which turned out to be an efficient route.

The accurate understanding of the risk factors and the outcome of the cardiac surgery, valvular surgery in particular, following prior pneumonectomy, have not been possible due to the very limited number of patients presented in the literature. In the review including both coronary artery bypass grafting and valve surgery patients by Stoller et al. [7], the overall mortality rate was $16 \%$ and wellexceeded valvular surgery in patients with preserved pulmonary functions. Only 5 to $8 \%$ of the mortality rate was attributed to the pulmonary complications. However, the perioperative respiratory failure was as high as $25 \%$ in the series comprising both coronary artery bypass grafting and valvular operations [7].

The surgical challenges in this group of patients in addition to high mortality/morbidity rates do not, however, preclude surgery. In appropriately selected cases, careful preoperative preparation aids in a favorable outcome. Short bypass times and short mechanical ventilation positively contributes to respiratory functions and prognosis.

\section{Acknowledgment}

Authors would like to thank Ms. Toni Spring for the linguistic revision of the paper. 


\section{References}

[1] F. Patanè, A. Verzini, E. Zingarelli, and M. di Summa, "Simultaneous operation for cardiac disease and lung cancer," Interactive Cardiovascular and Thoracic Surgery, vol. 1, no. 2, pp. 69-71, 2002.

[2] M. H. Danton, V. A. Anikin, K. G. McManus, J. A. McGuigan, and G. Campalani, "Simultaneous cardiac surgery with pulmonary resection: presentation of series and review of literature," European Journal of Cardiothoracic Surgery, vol. 13, no. 6, pp. 667-672, 1998.

[3] F. S. Vargas, M. Terra-Filho, W. Hueb, L. R. Teixeira, A. Cukier, and R. W. Light, "Pulmonary function after coronary artery bypass surgery," Respiratory Medicine, vol. 91, no. 10, pp. 629633, 1997.

[4] M. Hulusi Us, Y. Arslan, C. Özbek, M. Basaran, Y. Yildiz, T. Oğuş, and O. Işik, "Coronary artery bypass grafting after left pneumonectomy," Journal of Cardiothoracic and Vascular Anesthesia, vol. 20, no. 5, pp. 709-711, 2006.

[5] M. Ugurlucan, K. Nisli, E. Tireli, and E. Dayioglu, "Offpump total cavopulmonary connection in a patient with hypoplastic right pulmonary artery and right lung secondary to kyphoscoliosis," Journal of Cardiac Surgery, vol. 23, no. 5, pp. 565-568, 2008.

[6] J. A. Johnson, R. J. Landreneau, T. M. Boley, et al., "Should pulmonary lesions be resected at the time of open heart surgery?" American Surgeon, vol. 62, no. 4, pp. 300-303, 1996.

[7] J. K. Stoller, E. Blackstone, G. Pettersson, and T. Mihaljevic, "Coronary artery bypass graft and/or valvular operations following prior pneumonectomy: report of four new patients and review of the literature," Chest, vol. 132, no. 1, pp. 295-301, 2007.

[8] P. Giomarelli, S. Scolletta, E. Borrelli, and B. Biagioli, "Myocardial and lung injury after cardiopulmonary bypass: role of interleukin (IL)-10," Annals of Thoracic Surgery, vol. 76, no. 1, pp. 117-123, 2003. 


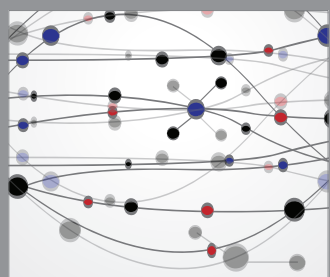

The Scientific World Journal
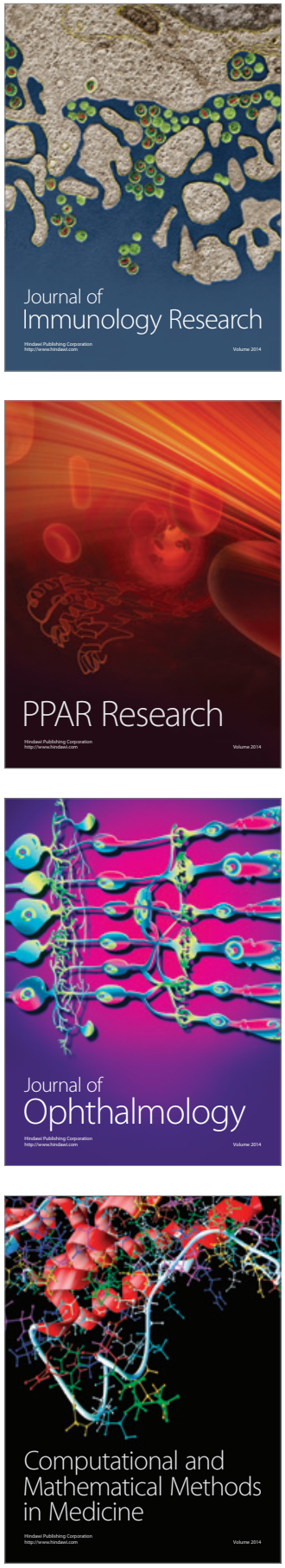

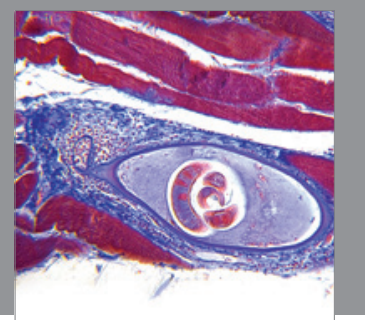

Gastroenterology

Research and Practice
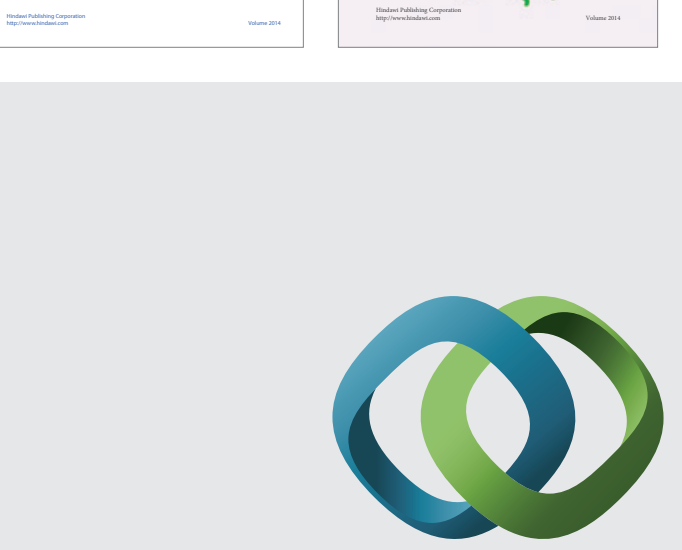

\section{Hindawi}

Submit your manuscripts at

http://www.hindawi.com
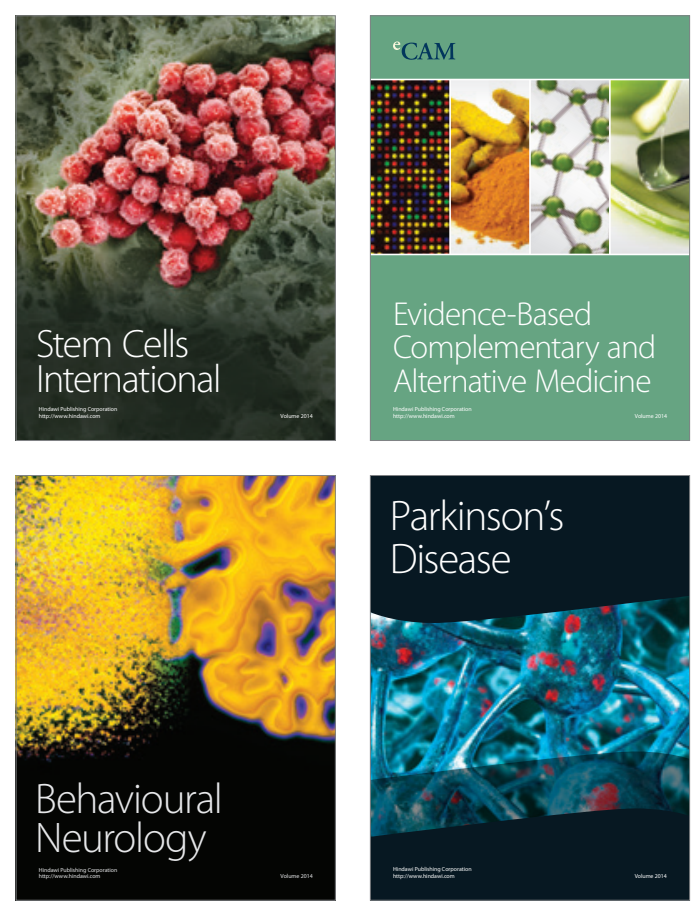

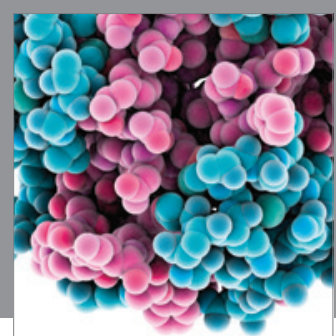

Journal of
Diabetes Research

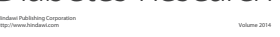

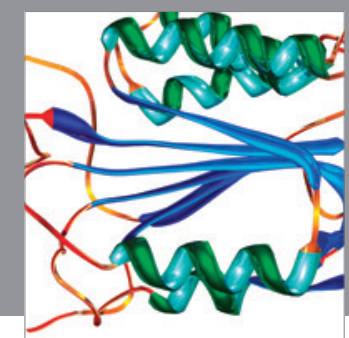

Disease Markers
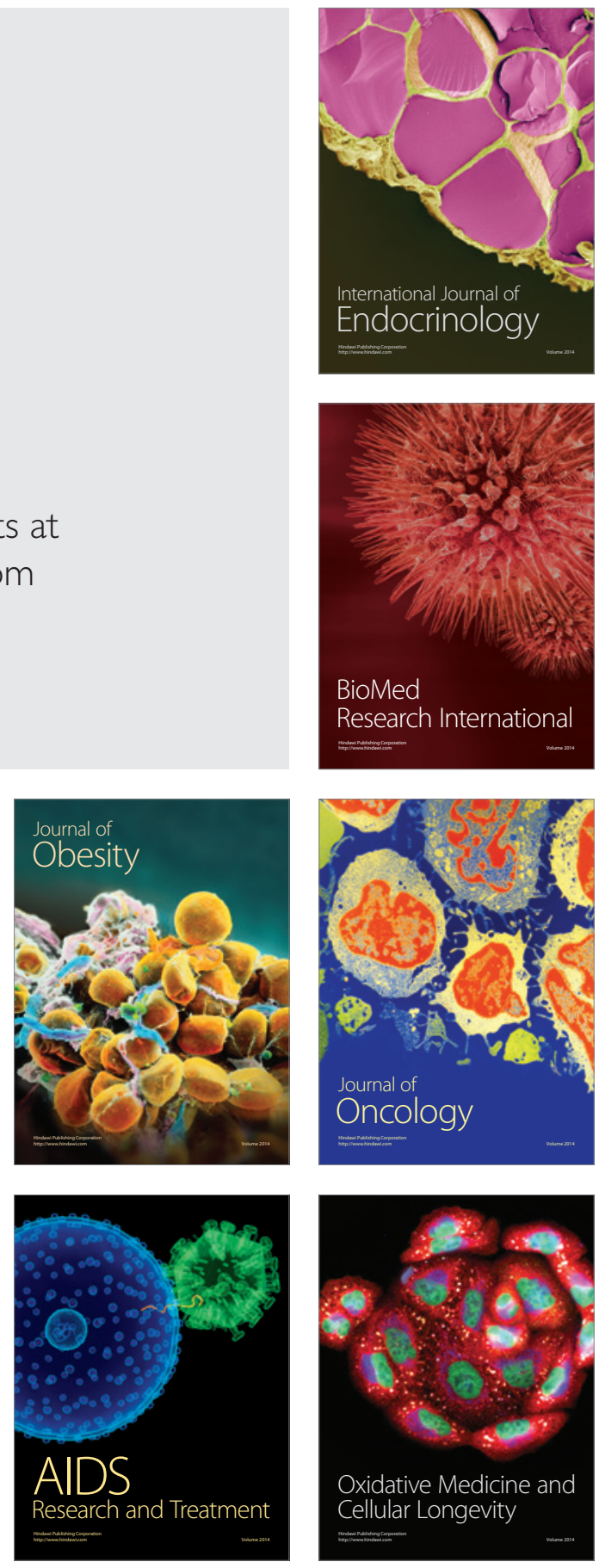